\title{
Histopathological alterations of the intrinsic tongue muscles following zoledronic acid treatment in a rat model
}

\section{Zmiany histopatologiczne wewnętrznych mięśni języka szczura po zastosowaniu kwasu zoledronowego}

\author{
Dina Badawy Farag ${ }^{A-F}$, Samah Sayed Mehanny ${ }^{A-F}$ \\ Department of Oral Biology, Faculty of Dentistry, Cairo University, Egypt \\ A - research concept and design; B - collection and/or assembly of data; $C$ - data analysis and interpretation; \\ $D$ - writing the article; $E$ - critical revision of the article; $F$ - final approval of the article
}

\section{Address for correspondence \\ Dina Badawy Farag}

E-mail: dina.badawyf@gmail.com

\section{Funding sources}

None declared

Conflict of interest

None declared

Received on 0ctober 18, 2019

Reviewed December 1, 2019

Accepted on December 16, 2019

Published online on May 28, 2020

Cite as

Farag DB, Mehanny SS. Histopathological alterations

of the intrinsic tongue muscles following zoledronic acid treatment in a rat model. Dent Med Probl. 2020;57(2):131-136. doi:10.17219/dmp/115368

DOI

$10.17219 / \mathrm{dmp} / 115368$

Copyright

(c) 2020 by Wroclaw Medical University

This is an article distributed under the terms of the

Creative Commons Attribution 3.0 Unported License (CC BY 3.0)

(https://creativecommons.org/licenses/by/3.0/).

\begin{abstract}
Background. Bisphosphonates (BPS) are widely used as anti-bone-resorptive agents. Despite the great benefits of BPs, they may cause local and systemic adverse side effects.

Objectives. The aim of this study was to evaluate the histopathological effect zoledronic acid (ZA), which belongs to $\mathrm{BPS}$, has on the intrinsic tongue muscles in a rat model.

Material and methods. A total of 30 adult male albino rats were divided into 3 groups (10 rats each): group I served as a control; group II was given an intraperitoneal (i.p.) injection of $0.2 \mathrm{mg} / \mathrm{kg}$ of ZA once per week for 3 weeks; and group III received the same dosage of ZA, but for 8 weeks. After the animals were euthanized, the tongue tissue was dissected and examined histologically, histochemically and immunohistochemically.
\end{abstract}

Results. Histologically, a normal architecture of the muscle fascicles was observed in the control group. Group II showed degenerated muscle fibers with an indistinct sarcolemma. In group III, the muscle fibers were degenerated with severe sarcoplasmic dissolution. The histochemical examination using Masson's trichrome (MT) demonstrated a significant increase in collagen fibers in groups II and III as compared to the control group. The immunohistochemical results revealed a statistically significantly higher expression of nuclear factor kappa B (NF-KB) in the ZA-treated groups (II and III) as compared to the control group, with the highest mean value recorded in group III.

Conclusions. Zoledronic acid induced histopathological changes to the intrinsic tongue muscles, and this effect was exaggerated with a longer duration of administration.

Key words: zoledronic acid, bisphosphonates, muscle fibers, collagen, rats

Słowa kluczowe: kwas zoledronowy, bisfosfoniany, włókna mię̧́niowe, kolagen, szzzury 


\section{Introduction}

Bisphosphonates (BPs) are a class of drugs that are widely used in the treatment of osteoclast-mediated bone loss. ${ }^{1}$ They are synthetic, stable analogs of inorganic pyrophosphates - naturally occurring polyphosphates found in urine and blood serum. The ability of BPs to bind divalent cations such as $\mathrm{Ca}^{2+}$ helps them bind to bone mineral surfaces, predominantly at sites of active bone remodeling. ${ }^{2,3}$ Bisphosphonates are usually classified into 2 main groups with different mechanisms of action: non-nitrogen-containing bisphosphonates (non-N-BPs) and nitrogen-containing bisphosphonates (N-BPs). ${ }^{4}$ Of all the antibone-resorptive BPs available nowadays, those containing nitrogen in the heterocyclic ring (zoledronic acid - ZA) are 10,000 times more potent than non-N-BPs. ${ }^{5}$

Zoledronic acid is well-established as a therapy for reducing skeletal-related events associated with bone metastases in several types of cancer, including prostate cancer. ${ }^{6}$ Moreover, ZA has been shown to improve immune surveillance against tumors, opening up new possibilities for therapeutic applications. ${ }^{7}$ However, several adverse effects have been demonstrated to be associated with ZA treatment. Bisphosphonate-associated osteonecrosis of the jaw ${ }^{8}$ and altered oral mucosal epithelium leading to delayed soft tissue healing ${ }^{9}$ are among the most significant side effects. An antiangiogenic effect of ZA has also been reported in many studies. It was observed that ZA could interfere with endothelial progenitor cell differentiation, ${ }^{10}$ impair endothelial cell proliferation, induce endothelial cell apoptosis, and modulate their adhesion and migration. $^{11}$

With this background in mind, the present study was performed to evaluate the possible effect of ZA treatment on the intrinsic tongue muscles in male albino rats using histological, histochemical and immunohistochemical analysis.

\section{Material and methods}

\section{Ethical statement}

This experimental study was carried out in the animal house of the Faculty of Medicine at Cairo University in Egypt, according to the recommendations and approval of the Institutional Animal Care and Use Committee of Cairo University (CU-IACUC) (approval No. CU/III/F/37/19).

\section{Experimental design}

A total of 30 adult male albino Wistar rats weighing $200 \pm 10 \mathrm{~g}$ were included in the study. All animals were housed in a sterile environment, maintained at room temperature $\left(21 \pm 2^{\circ} \mathrm{C}\right)$ and $50-55 \%$ humidity with a 12 -hour light cycle. The animals were fed with pelleted rat food and water ad libitum. The rats were randomly divided into 3 equal groups, with 10 rats in each group. The control group (group I) rats were given an intraperitoneal (i.p.) injection of saline solution. The group II rats received $0.2 \mathrm{mg} / \mathrm{kg}$ of ZA ${ }^{12}$ (Zometa $^{\circledR}$; Novartis, Basel, Switzerland) i.p. once per week for 3 weeks. The rats in group III received $0.2 \mathrm{mg} / \mathrm{kg}$ of $\mathrm{ZA}^{12}$ i.p. once per week for 8 weeks. All animals were euthanized by an i.p. injection of anesthetic overdose and the tongue was dissected.

\section{Light microscopic examination}

All tongue specimens were fixed in $10 \%$ formaldehyde solution. After staying in a fixative material for $24 \mathrm{~h}$, the samples were washed under running water, dehydrated in an ethanol series, then cleared in xylene, and embedded in liquid paraffin. The tissues were then cut into 4-6-micrometer-thick sections and subjected to examination.

\section{Histopathological examination}

The sections were stained with hematoxylin and eosin (H\&E).

\section{Histochemical examination}

The sections were stained with Masson's trichrome (MT) for the detection of collagen fibers.

\section{Immunohistochemical examination}

The sections were deparaffinized and hydrated, then washed in $0.1 \mathrm{M}$ phosphate buffer saline (PBS). Endogenous peroxidases were blocked by treatment with $\mathrm{H}_{2} \mathrm{O}_{2}$ in methanol. Non-specific background staining was inhibited through incubation at room temperature for $30 \mathrm{~min}$ in $0.3 \%$ bovine serum albumin. The sections were incubated with primary antibodies for nuclear factor kappa B (NF-kB) at room temperature for $60 \mathrm{~min}$. Then, the sections were washed in buffer 3 times, each time for $5 \mathrm{~min}$, and incubated for further $30 \mathrm{~min}$ with biotinylated secondary antibodies, followed by washing. Diaminobenzidine solution was used as a chromogen and Mayer's hematoxylin was used as a counterstain for $5 \mathrm{~min}$. Phosphate buffer saline was used as a negative controller. The slides were then finally mounted. Positive immunoreactivity for NF- $\mathrm{KB}$ appeared in the form of brown coloration of the cytoplasm and/or nuclei of the immunoreactive cells.

\section{Image analysis}

For the evaluation of staining affinity, the area percentage of collagen fibers in the sections stained with MT as well as NF-kB immunoreactivity were measured 
by an image analyzer (Leica DM LB2 with QWin Plus image analysis software; Leica Camera, Wetzlar, Germany). The image analysis was done in different, non-overlapping fields of each specimen.

\section{Statistical analysis}

The data obtained from the image analysis was statistically described in terms of mean \pm standard deviation $(M \pm S D)$. Student's $t$-test was used for multiple pairwise comparisons. A probability value ( $p$-value) of $<0.05$ was considered statistically significant. All statistical calculations were done using Microsoft Excel 2007 (Microsoft Corporation, Redmond, USA) and SPSS for Windows v. 15 (SPSS Inc., Chicago, USA).

\section{Results}

\section{Histopathological results}

Group I (the control group) showed a normal architecture of the muscle fascicles. The muscle fibers were arranged differently, with some being cut longitudinally while others were cut transversely. The fibers demonstrated homogenous acidophilic sarcoplasm with multiple peripheral elongated nuclei beneath a well-defined sarcolemma (Fig. 1A).

The muscle fascicles in group II were ill-defined as compared to the control group. Degenerated muscle fibers with an indistinct sarcolemma were also detected. Degeneration was in the form of serration, widely distributed sarcoplasmic dissolution and nuclear atypia (change in size and position). Chronic inflammatory cellular infiltrate was obvious (Fig. 1B).
Group III demonstrated the degeneration of most of the intrinsic muscle fibers of the tongue. Degenerated muscle fibers appeared swollen with severe sarcoplasmic dissolution. The remnants of eosinophilic muscle fibers were also observed within the coalesced fascicles. A thickened perimysium with marked inflammatory cell inflitration was noticed. Histiocytes appeared with different patterns: with homogenous basophilic cytoplasm, with a central basophilic body, or with a regular pattern of giant, deeply basophilic stained cells. The accumulation of fat droplets was also noted (Fig. 1C).

\section{Histochemical results}

The histochemical examination using MT for the detection of collagen fiber bundles (the stains in blue) revealed that the tongue intrinsic muscles of the control group consisted of tightly packed, regularly arranged, parallel perimysial collagen bundles (Fig. 2A). The mean collagen area percentage was $7.06 \pm 0.21$.

Disorganized perimysial collagen fiber bundles were observed in group II. Some areas showed dense, wavy collagen bundles related to clumped muscle fascicles. However, other areas had a thin perimysium (Fig. 2B). The mean value of collagen area percentage was $17.77 \pm 2.19$; statistical analysis revealed that it was significantly higher than that of group I $(p=0.015)$.

Group III exhibited dense, curly, disorganized perimysial collagen bundles (Fig. 2C). The highest mean value of collagen area percentage was found in this group $-23.55 \pm 1.11$. Statistical analysis revealed that this mean value was statistically significantly higher as compared to group I ( $p=0.004)$, but it was not statistically significantly different from that of group II $(p=0.081)$.

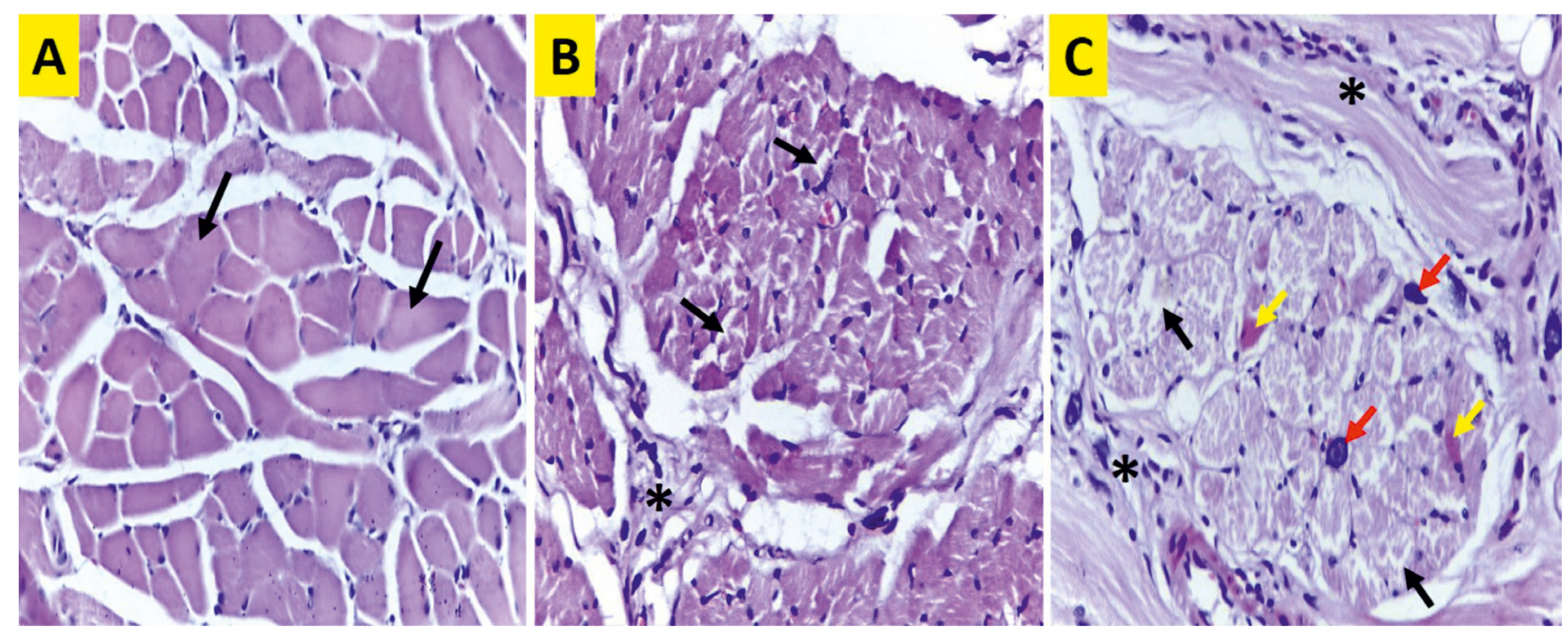

Fig. 1. Photomicrograph of the intrinsic tongue muscles (hematoxylin and eosin (H\&E) staining)

A - control group with normally arranged muscle fibers (arrows) and a well-defined sarcolemma; B - group II with degenerated muscle fibers (arrows) and chronic inflammatory cell infiltration (asterisk); C - group III with severe muscle fiber degeneration (black arrows), eosinophilic coagulation (yellow arrows), a thickened perimysium with chronic inflammatory cells (asterisks), and histiocytes (red arrows); original magnification $\times 400$. 

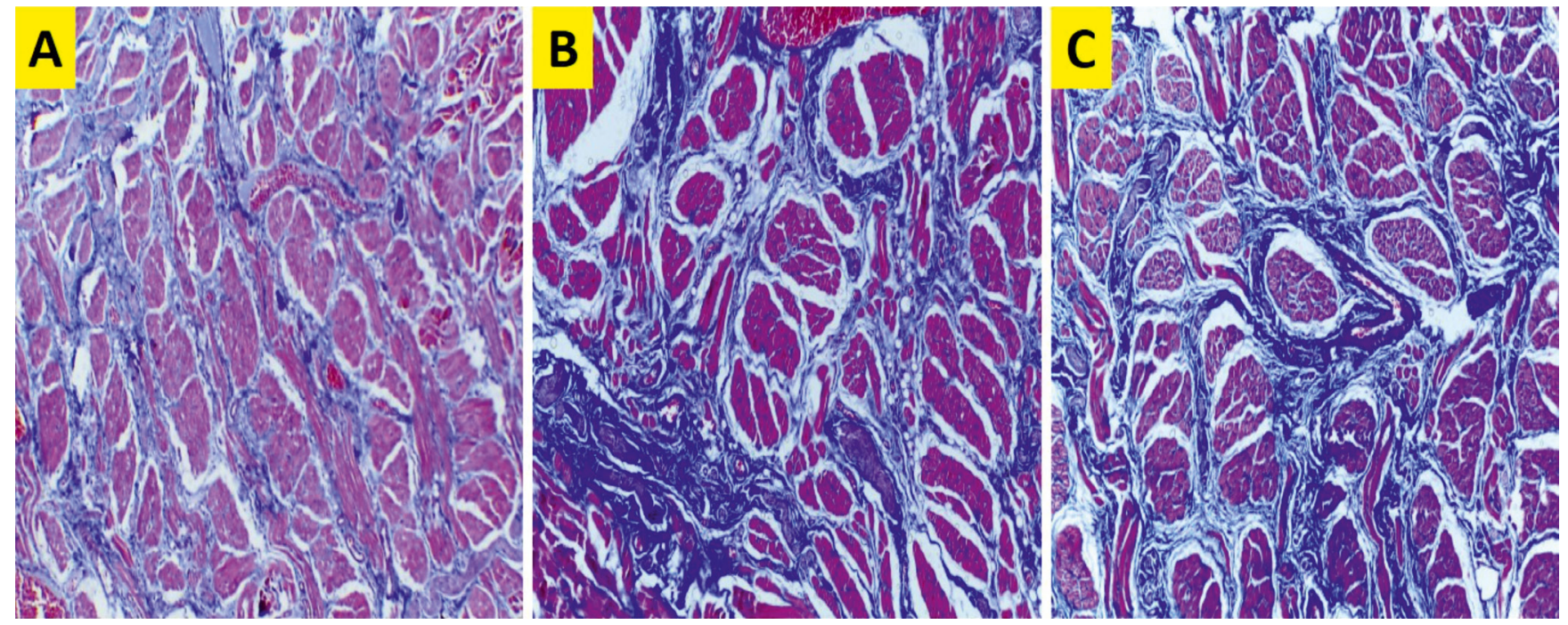

Fig. 2. Photomicrograph of the intrinsic tongue muscles (Masson's trichrome (MT) staining)

A - control group with regularly arranged collagen fibers in the perimysium; B - group II with disorganized, wavy perimysial collagen fibers; C - group III with disorganized, curly perimysial collagen fibers; original magnification $\times 100$.

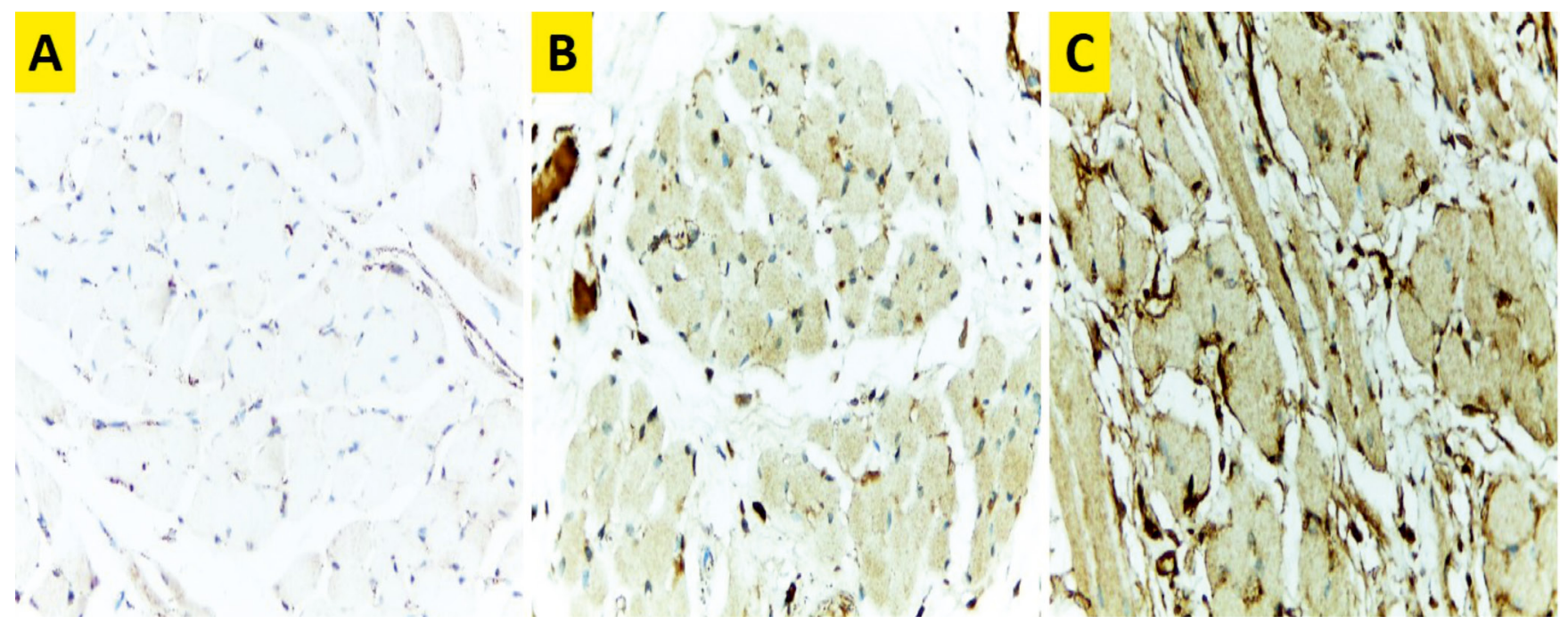

Fig. 3. Photomicrograph of the intrinsic tongue muscles showing (nuclear factor kappa B (NF-kB) staining)

A - negative immunoreactivity for activated NF-KB in the control group; B - moderate immunoreactivity for activated NF-KB in the cytoplasm and nucleus of the muscle fibers in group II; C - high immunoreactivity for activated NF-KB in the cytoplasm and nucleus of the muscle fibers in group III; original magnification $\times 400$.

\section{Immunohistochemical results}

The NF- $k B$ immunohistochemical staining of the intrinsic tongue muscle tissue from the control group showed no detectable cytoplasmic or nuclear immunoreactivity (Fig. 3A). The mean percentage of the area of NF- $\mathrm{kB}$ immunoexpression in this group was $0.06 \pm 0.03$.

Moderate immunoreactivity for NF- $\mathrm{kB}$ was observed in the cytoplasm of the muscle fibers in group II. Some immunopositive muscle fibers showed positively stained nuclei (Fig. 3B). The mean percentage of the area of NF- $\mathrm{kB}$ immunoexpression was $22.74 \pm 4.18$. Statistical analysis revealed that this mean value was significantly higher as compared to group I $(p=0.000)$.
High cytoplasmic and nuclear immunoreactivity for activated NF- $\mathrm{kB}$ was noted in group III (Fig. 3C). The highest mean percentage of the area of NF- $\mathrm{kB}$ immunoexpression was revealed in this group $-36.32 \pm 2.56$. Statistical analysis revealed that this value was statistically significantly different from that of both group I $(p=0.000)$ and group II $(p=0.003)$.

\section{Discussion}

Zoledronic acid is a highly effective drug that inhibits osteoclast-mediated bone resorption. ${ }^{13}$ Although ZA is well-tolerated, numerous short-term and long-term adverse reactions have been reported to be associated with 
the administration of ZA. ${ }^{14}$ Severe musculoskeletal pain is one of the side effects related to BP treatment. It has been reported to occur days or months (median time: 14 days) after starting a BP therapy and to resolve only if the therapy is stopped. ${ }^{15}$ Based on this clinical observation, the skeletal muscle was the tissue of choice to be investigated in the current study.

The tongue is a unique skeletal muscle with differences in the muscle fiber composition when compared with limb, masticatory and orofacial muscles, most likely reflecting genotypic and phenotypic functional specialization in oral function. The prevalence of type II fibers, regional differences in the fiber composition and complex muscle structure generally suggest rapid and flexible actions in shaping and positioning the tongue while performing vital functions, such as speech, swallowing, mastication, and breathing. ${ }^{16}$ These facts reinforce the decision to select the intrinsic tongue muscles to be the examined tissue in this study.

The histopathological examination in the present study revealed a normal architecture of the muscle fascicles in the control group. On the other hand, group II (3 weeks of ZA treatment) showed degenerated muscle fibers with an indistinct sarcolemma. Swollen muscle fibers with severe sarcoplasmic dissolution were observed after 8 weeks of ZA administration (group III). Inflammatory cellular infiltration was noticed in both ZA-treated groups. Interestingly, the muscle tissue in the group III rats was infiltrated by various patterns of histiocytes. Cells with central basophilic bodies was one of the patterns observed in the present study. Histologically, as explained by Gillett et al., ${ }^{17}$ this pattern is a result of defective phagolysosomal activity within macrophages; thus, it will contain partially digested bacteria. This leads to the deposition of calcium and iron, resulting in a basophilic inclusion structure (Michaelis-Gutmann bodies). Large macrophages which are present at sites of infection (von Hansemann cells) exhibit numerous secondary lysosomes containing partially digested organisms. The fusion and calcification of these lysosomes result in the formation of intracytoplasmic bodies called Michaelis-Gutmann bodies. It has been proposed that local bacterial antigen load, due to tissue necrosis, might lead to the accumulation of macrophages, which would facilitate the local production of Michaelis -Gutmann bodies. ${ }^{18}$ Such an explanation could be supported by the current study, suggesting extensive muscle degeneration in group III. Another postulate could be that bacterial invasion may occur as a result of the negative effect of BPs on the adhesion and metabolism of oral mucosal cells. ${ }^{19}$

The degeneration and inflammatory cellular infiltration associated with ZA treatment observed in this study supports previous studies, which reported obvious histopathological changes following ZA administration. These changes included severe tubular degeneration, hypereosinophilia, cell necrosis, and interstitial fibrosis in the renal tissue. ${ }^{20,21}$ Furthermore, necrotic zones and intense acute inflammatory infiltrate were observed in the alveolar bone tissue of rats receiving $\mathrm{ZA} .^{12}$

The NF- $\mathrm{kB}$ complex is activated in response to a variety of stimuli, including bacterial infection, exposure to proinflammatory cytokines and growth factors, and oxidative and biomechanical stress. ${ }^{22}$ In this study, activated NF- $\mathrm{kB}$ was immunohistochemically located in the tongue muscle tissue as an indicator of an inflammatory response and oxidative stress. The results showed that NF- $\mathrm{kB}$ immunoexpression was higher following ZA administration, with the greatest mean value recorded after 8 weeks (group III). This was in accordance with Muratsu et al., who revealed that $\mathrm{ZA}$ activated NF- $\mathrm{kB}$ expression in a cultured murine macrophage cell line. ${ }^{23}$

The correlation between the histopathological and immunohistochemical results observed in the present study could be explained by understanding the ZA molecular mechanism of action. Zoledronic acid has been reported to be the most potent N-BP in inhibiting the enzymes farnesyl diphosphate synthase (FPPS) and geranylgeranyl diphosphate synthase (GGPPS) in the mevalonate pathway. ${ }^{24}$

The inhibition of FPPS has been suggested to increase the intracellular levels of isopentyl pyrophosphate, which induces T-cell activation; this results in the release of inflammatory cytokines. ${ }^{15}$ It has also been reported that the inactivation of GGPPS results in the stimulation of the proinflammatory mitogen-activated protein kinases and NF- $\mathrm{B}$ signaling pathways. ${ }^{25}$ Although NF- $\mathrm{kB}$ regulates the expression of proinflammatory cytokines, these cytokines are considered potent activators of NF- $\mathrm{kB}$. This establishes a positive feedback loop, resulting in the overstimulation of NF- $\mathrm{kB} .{ }^{22}$ It has been suggested that the persistent stimulation of the skeletal muscle fibers through positive feedback loops may result in the overstimulation of NF-kB. ${ }^{26}$ This hypothesis could explain the enhanced NF- $\mathrm{kB}$ immunoexpression observed in the present study. Nuclear factor kappa B has been reported to be one of the most important signaling pathways related to the loss of the skeletal muscle mass. The activation of NF- $\mathrm{kB}$ in the skeletal muscle leads to the degradation of specific muscle proteins, induces inflammation and fibrosis, and blocks the regeneration of myofibers after injury/atrophy. ${ }^{27}$

The MT staining procedure was used in the present study to assess the extent of fibrosis ${ }^{28}$ in the intrinsic tongue muscles. A progressive increase in the amount of collagen fibers was observed among the experimental groups. The lowest mean value was recorded in the control group, whereas the highest value was recorded in group III. An increase in fibrosis was concomitant with a degree of muscle injury.

According to Lańcut et al., an increase in the intrafasicular connective tissue usually represents a response to myofiber loss, wherein fibroblasts replace the damaged area, with the subsequent formation of collagen fibers. ${ }^{29}$ 
It has also been suggested that a persistent inflammatory response alters the extracellular environment and increases the secretion of various inflammatory cytokines, which contributes to muscle fibrosis. ${ }^{30}$

\section{Conclusions}

It can be concluded that ZA induces histopathological changes in the intrinsic tongue muscles, and that this effect is exaggerated by a longer administration. These changes might predict a poorer functional outcome of these muscles.

\section{Recommendations}

Since the intrinsic tongue muscles contribute substantially to chewing, swallowing, speaking, and respiration, it is recommended that clinicians be aware of the expected poor outcomes in these functions in patients under ZA treatment. Further studies need to be performed on other muscles of the aerodigestive tract involved in swallowing and mastication, as well as on females, using various investigatory tools.

\section{ORCID iDs}

Dina Badawy Farag (1) https://orcid.org/0000-0002-8856-8906 Samah Sayed Mehanny (1) https://orcid.org/0000-0002-8910-904X

\section{References}

1. Shaw NJ, Bishop NJ. Bisphosphonate treatment of bone disease. Arch Dis Child. 2005;90(5):494-499.

2. Rogers MJ. New insights into the molecular mechanisms of action of bisphosphonates. Curr Pharm Des. 2003;9(32):2643-2658.

3. Masarachia P, Weinreb M, Balena R, Rodan GA. Comparison of the distribution of $3 \mathrm{H}$-alendronate and $3 \mathrm{H}$-etidronate in rat and mouse bones. Bone. 1996;19(3):281-290.

4. Lehenkari PP, Kellinsalmi $M$, Näpänkangas JP, et al. Further insight into mechanism of action of clodronate: Inhibition of mitochondrial ADP/ATP translocase by a nonhydrolyzable, adenine-containing metabolite. Mol Pharmacol. 2002;61(5):1255-1262.

5. Ballantyne E. Bisphosphonates: Possible modes of action and implications for dental implant treatment. A review of the literature. J Gen Pract. 2015;3:192.

6. Valdespino V, Tsagozis P, Pisa P. Current perspectives in the treatment of advanced prostate cancer. Med Oncol. 2007;24(3):273-286.

7. Coscia M, Quaglino E, lezzi $M$, et al. Zoledronic acid repolarizes tumour-associated macrophages and inhibits mammary carcinogenesis by targeting the mevalonate pathway. J Cell Mol Med. 2010;14(12):2803-2815.

8. Ruggiero SL. Bisphosphonate-related osteonecrosis of the jaw: An overview. Ann N Y Acad Sci. 2011;1218:38-46.

9. Allam E, Allen $M$, Chu TM, Ghoneima A, Jack Windsor L. In vivo effects of zoledronic acid on oral mucosal epithelial cells. Oral Dis. 2011;17(3):291-297.

10. Yamada J, Tsuno NH, Kitayama J, et al. Anti-angiogenic property of zoledronic acid by inhibition of endothelial progenitor cell differentiation. J Surg Res. 2009;151(1):115-120.

11. Wood J, Bonjean K, Ruetz S, et al. Novel antiangiogenic effects of the bisphosphonate compound zoledronic acid. J Pharmacol Exp Ther. 2002;302(3):1055-1061.

12. Pacheco VN, Langie R, Benfica JRD, et al. Nitrogen-containing bisphosphonate therapy - Part II: Assessment of alveolar bone tissue inflammatory response in rats - a blind randomized controlled trial. Int J Exp Pathol. 2018;99(5):258-263.
13. Berenson JR, Rosen LS, Howell A, et al. Zoledronic acid reduces skeletal-related events in patients with osteolytic metastases. Cancer. 2001;91(7):1191-1200.

14. Kuchay MS, Farooqui KJ, Mithal A. Acute severe diarrhea and hyponatremia after zoledronic acid infusion: An acute phase reaction. Clin Cases Miner Bone Metab. 2017;14(1):101-104.

15. Lim SY, Bolster MB. What can we do about musculoskeletal pain from bisphosphonates? Cleve Clin J Med. 2018;85(9):675-678.

16. Stål P, Marklund S, Thornell LE, De Paul R, Eriksson PO. Fibre composition of human intrinsic tongue muscles. Cells Tissues Organs. 2003;173(3):147-161.

17. Gillett MB, Pradeep KE, Mikhail M. Malacoplakia of the tongue. J Clin Pathol. 2006;59(1):112.

18. Pesce C, Pate G, Valente S, Tanzi R. Focal malakoplakia in chronic periapical periodontitis. Histopathology. 1999;34(2):140-143.

19. Basso FG, Pansani TN, Soares DG, Cardoso LM, Hebling J, de Souza Costa CA. Influence of bisphosphonates on the adherence and metabolism of epithelial cells and gingival fibroblasts to titanium surfaces. Clin Oral Investig. 2018;22(2):893-900.

20. Markowitz GS, Fine PL, Stack Jl, et al. Toxic acute tubular necrosis following treatment with zoledronate (Zometa). Kidney Int. 2003;64(1):281-289.

21. Pfister T, Atzpodien E, Bauss F. The renal effects of minimally nephrotoxic doses of ibandronate and zoledronate following single and intermittent intravenous administration in rats. Toxicology. 2003;191(2-3):159-167.

22. Kumar A, Takada Y, Boriek AM, Aggarwal BB. Nuclear factor-kappaB: Its role in health and disease. J Mol Med (Berl). 2004;82(7):434-448.

23. Muratsu D, Yoshiga D, Taketomi T, et al. Zoledronic acid enhances lipopolysaccharide-stimulated proinflammatory reactions through controlled expression of SOCS1 in macrophages. PLoS One. 2013;8(7):e67906.

24. Gong L, Altman RB, Klein TE. Bisphosphonates pathway. Pharmacogenet Genomics. 2011;21(1):50-53.

25. Wang $X X$, Ying $P$, Diao $F$, et al. Altered protein prenylation in Sertoli cells is associated with adult infertility resulting from childhood mumps infection. J Exp Med. 2013;210(8):1559-1574.

26. Kumar A, Boriek AM. Mechanical stress activates the nuclear factorkappaB pathway in skeletal muscle fibers: A possible role in Duchenne muscular dystrophy. FASEB J. 2003;17(3):386-396.

27. Thoma A, Lightfoot AP. NF-KB and inflammatory cytokine signaling: Role in skeletal muscle atrophy. Adv Exp Med Biol. 2018;1088:267-279.

28. Meinen S, Barzaghi P, Lin S, Lochmüller H, Ruegg MA. Linker molecules between laminins and dystroglycan ameliorate lamininalpha2-deficient muscular dystrophy at all disease stages. J Cell Biol. 2007;176(7):979-993.

29. Lańcut M, Jedrych B, Lis-Sochocka M, Czerny K. Histological and ultrastructural changes in cross-striation muscle cells, under the influence of atorvastatin-reductase HMG-CoA inhibitor. Ann Univ Mariae Curie Sklodowska Med. 2004;59(2):32-37.

30. Gosselin LE, McCormick KM. Targeting the immune system to improve ventilatory function in muscular dystrophy. Med Sci Sports Exerc. 2004;36(1):44-51. 